\title{
FAST RADIO BURST/GAMMA-RAY BURST COSMOGRAPHY
}

\author{
He $\mathrm{GAO}^{1}$, Zhuo $\mathrm{Li}^{2,3}$, Bing ZHANG ${ }^{1,2,3}$ \\ ${ }^{1}$ Department of Physics and Astronomy, University of Nevada Las Vegas, NV 89154, \\ USA;gaohe@physics.unlv.edu; zhang@physics.unlv.edu \\ ${ }^{2}$ Department of Astronomy, School of Physics, Peking University, Beijing 100871, China; zhuo.li@pku.edu.cn \\ ${ }^{3}$ Kavli Institute of Astronomy and Astrophysics, Peking University, Beijing 100871, China
}

\begin{abstract}
Recently, both theoretical arguments and observational evidence suggested that a small fraction of fast radio bursts (FRBs) could be associated with gamma-ray bursts (GRBs). If such FRB/GRB association systems are commonly detected in the future, the combination of dispersion measure (DM) derived from FRBs and redshifts derived from GRBs makes these systems a plausible tool to conduct cosmography. We quantify uncertainties in deriving the redshift-dependent $\mathrm{DM}_{\mathrm{IGM}}$ as a function of $z$, and test how well dark energy models can be constrained with Monte Carlo simulations. We show that with potentially several 10s of FRB/GRB systems detected in a decade or so, one may reach reasonable constraints on $w$ CDM models. When combined with SN Ia data, unprecedented constraints on dark energy equation of state may be achieved, thanks to the prospects of detecting FRB/GRB systems at relatively high redshifts. The ratio between the mean value $\left\langle\operatorname{DM}_{\operatorname{IGM}}(z)\right\rangle$ and luminosity distance $\left(D_{\mathrm{L}}(z)\right)$ is insensitive to dark energy models. This gives the prospects of applying SN Ia data to calibrate $\left\langle\mathrm{DM}_{\mathrm{IGM}}(z)\right\rangle$ using a relatively small sample of FRB/GRB systems, allowing a reliable constraint on the baryon inhomogeneity distribution as a function of redshift. The methodology developed in this paper can also be applied, if the FRB redshifts can be measured by other means. Some caveats of putting this method into practice are also discussed.
\end{abstract}

Subject headings:

\section{INTRODUCTION}

The nature of late time cosmic acceleration is a deep mystery in cosmology and fundamental physics, which could be explained by introducing an exotic form of energy content with negative pressure, dubbed dark energy. Cosmological parameters have been measured via various standard candles or rulers, such as Type Ia supernovae (SN Ia) (Riess et al. 1998), baryon acoustic oscillations (BAO) (Anderson et al. 2012; Beutler et al. 2011), as well as small scale anisotropies of the cosmic microwave background (CMB) radiation (Hinshaw et al. 2013; Ade et al. 2013). Being bright beacons from deep universe, gamma-ray bursts (GRBs) have been considered as a potential complementary probe to conduct cosmography. Many authors have made use of GRB luminosity indicators as standard candles (e.g. Dai et al. 2004; Ghirlanda et al. 2004; Liang \& Zhang 2005; Schaefer 2007). However, unlike the SN Ia candle, GRB correlations lack physical motivation and usually have relatively large scatter, so that their role as standard candles is debated. Nonetheless, GRBs can serve as a complementary tool to probe the relatively high- $z$ universe, such as the star formation history, the metal enrichment history, and the properties of intergalactic medium (e.g. Barkana \& Loeb 2004; McQuinn et al. 2008; Virgili et al.|2011; Wang et al. 2012).

Recently, Thornton et al. (2013) reported the discovery of a new type of cosmological transients, dubbed Fast Radio Bursts (FRBs). These objects have anomalously high dispersion measure (DM) values corresponding to a cosmological redshift between 0.5 and 1 (Lorimer et al.
2007; Thornton et al. 2013) ${ }^{1}$. If the redshifts of these events can be measured, the combination of $z$ and DM information would be invaluable to conduct cosmography. Zhang (2014) suggested that a small fraction of FRBs could be physically connected to some GRBs, whose central engine is a supra-massive millisecond magnetar which collapses to a black hole at $10^{2}-10^{4}$ seconds after the burst ${ }^{2}$. Two possible such associations might have been observed by Bannister et al. (2012), and the fraction of GRBs that might host a magnetar central engine, and hence, a possible FRB, could be up to $\sim 60 \%$ for long GRBs (Lü \& Zhang 2014) and probably an even higher fraction for short GRBs (Rowlinson et al. 2013). Using the dispersion measure (DM) values of the two possible FRB/GRB association candidates (Bannister et al. 2012), Deng \& Zhang (2014) derived the upper limits of the baryon mass density along the line-of-sight of the two GRBs, which are consistent with the values derived by other methods. This lends further support to FRB/GRB associations. The FRB/GRB association systems, if commonly detected in the future, would be an ideal tool to constrain cosmological parameters and properties of dark energy at redshifts not attainable by SN Ia. We term this prospect as "FRB/GRB cosmography".

In this paper, we study the prospects of conducting FRB/GRB cosmography in detail. An independent work was recently carried out by Zhou et al. (2014), who discussed using FRBs to constrain dark energy properties

1 Some recent works also invoke a galactic origin for some of the FRBs (Loeb et al. 2014; Bannister \& Madsen 2014; Kulkarni et al. 2014).

2 Most FRBs would be produced by supra-massive NSs collapsing into a black hole after a much longer delay, e.g. thousands to millions of years (Falcke \& Rezzolla 2014). 
assuming that the redshifts of FRBs can be measured.

\section{UNCERTAINTIES IN INTERGALACTIC MEDIUM DM VALUE ESTIMATION}

For an FRB/GRB system, the measured dispersion measure (Deng \& Zhang 2014)

$$
\mathrm{DM}_{\mathrm{obs}}=\mathrm{DM}_{\mathrm{MW}}+\mathrm{DM}_{\mathrm{IGM}}+\mathrm{DM}_{\text {Host }}+\mathrm{DM}_{\mathrm{GRB}}
$$

has contributions from the Milky Way, intergalactic medium, GRB host galaxy, and the GRB blastwave, respectively. Among these terms,

$$
\begin{aligned}
\mathrm{DM}_{\mathrm{IGM}} & =\mathrm{DM}_{\mathrm{obs}}-\left(\mathrm{DM}_{\mathrm{MW}}+\mathrm{DM}_{\mathrm{GRB}}+\mathrm{DM}_{\text {Host }}\right) \\
& =\left\langle\mathrm{DM}_{\mathrm{IGM}}\right\rangle+\Delta\left(\mathrm{DM}_{\mathrm{IGM}}\right)
\end{aligned}
$$

is the relevant one to probe the universe. Here $\left\langle\mathrm{DM}_{\mathrm{IGM}}\right\rangle(z)$ is the IGM DM averaged in all directions for a given $z$, which is defined by cosmological parameters. By introducing the fraction of ionized electrons in hydrogen $(\mathrm{H})$ and helium $(\mathrm{He})$ atoms as a function of redshift $\left(\chi_{\mathrm{e}, \mathrm{H}}(z)\right.$ and $\left.\chi_{\mathrm{e}, \mathrm{He}}(z)\right)$, and assuming $\mathrm{H}: \mathrm{He}$ mass ratio is approximately $3: 1$, one can give a general expression for $\left\langle\mathrm{DM}_{\mathrm{IGM}}\right\rangle$ by generalizing Eq.(13) of Deng \& Zhang (2014):

$$
\left\langle\operatorname{DM}_{\mathrm{IGM}}(z)\right\rangle=\frac{3 c H_{0} \Omega_{b} f_{\mathrm{IGM}}}{8 \pi G m_{p}} \int_{0}^{z} \frac{\chi\left(z^{\prime}\right)\left(1+z^{\prime}\right) d z^{\prime}}{E\left(z^{\prime}\right)} .
$$

where

$$
\begin{aligned}
& \chi(z)=\frac{3}{4} y_{1} \chi_{\mathrm{e}, \mathrm{H}}(z)+\frac{1}{8} y_{2} \chi_{\mathrm{e}, \mathrm{He}}(z), \\
& E(z)=\left[(1+z)^{3} \Omega_{\mathrm{M}}+f(z) \Omega_{\mathrm{DE}}+(1+z)^{2} \Omega_{\mathrm{k}}\right]^{1 / 2}, \\
& f(z)=\exp \left[3 \int_{0}^{z} \frac{\left(1+w\left(z^{\prime \prime}\right)\right) d z^{\prime \prime}}{\left(1+z^{\prime \prime}\right)}\right],
\end{aligned}
$$

$\Omega_{b}$ is the current baryon mass fraction of the universe, $f_{\text {IGM }}$ is the fraction of baryon mass in the intergalactic medium, and $y_{1} \sim 1$ and $y_{2} \sim 1$ are IGM hydrogen and helium mass fractions normalized to $3 / 4$ and $1 / 4$, respectively. The term $\Delta\left(\mathrm{DM}_{\mathrm{IGM}}\right)$ (can be both positive and negative) in Eq.(2) stands for deviation DM IGM $_{\text {from }}$ the mean value at individual lines of sight due to the inhomogeneity of the baryon matter in the universe (e.g. McQuinn 2014).

Many dark energy models invoke $w \neq-1$, and many have $w(z)$ not a constant. Ideally (if $\Delta \mathrm{DM}_{\mathrm{IGM}}$ is not large), these models may be differentiated with a good sample of FRB/GRB systems spreading in a wide redshift range, as long as one could precisely measure $\mathrm{DM}_{\mathrm{obs}}$ and precisely determine $\mathrm{DM}_{\mathrm{MW}}+\mathrm{DM}_{\text {Host }}+\mathrm{DM}_{\mathrm{GRB}}$. The advantage of using $\left\langle\mathrm{DM}_{\mathrm{IGM}}\right\rangle$ to conduct cosmography is that the underlying physics is clean, which stems from the simple geometry of the universe, in constrast to other standard candles or rulers that invoke messier physics (e.g. SN Ia candle relies on poorly known supernvoa explosion physics). In the following, we discuss how to practically determine various DM components and estimate their relevant uncertainties in turn.

- The measurement of $\mathrm{DM}_{\mathrm{obs}}$ is very accurate, for instance, the uncertainties for the four reported FRBs are $0.05,0.3,0.7$ and $0.3 \mathrm{pc} \mathrm{cm}^{-3}$ respectively. Here we use an average of these four values to estimate the uncertainty of $\mathrm{DM}_{\mathrm{obs}}$, i.e, $\sigma_{\mathrm{obs}}=$ $0.34 \mathrm{pc} \mathrm{cm}^{-3}$, which is negligible compared with other uncertainties.
- $\mathrm{DM}_{\mathrm{MW}}$ can be estimated to within a factor of 1.5-2 using Galactic pulsar data (Taylor \& Cordes 1993). It rapidly drops to small values as the Galactic latitude is $|b|>10^{\circ}$. We therefore suggest to take this condition as our sample selection criterion in the future. With the ATNF pulsar data ${ }^{3}$ (Manchester et al. 2005), we find that the average dispersion of $\mathrm{DM}_{\mathrm{MW}}$ for $|b|>10^{\circ}$ sources is 33 pc $\mathrm{cm}^{-3}$ (see Fig. 1a), and we take this value as $\sigma_{\mathrm{MW}}$.

- In principle, one could precisely calculate $\mathrm{DM}_{\mathrm{GRB}}$ based on the GRB afterglow models if the model parameters could be constrained. Deng \& Zhang (2014) presented some calculation results given typical parameters and found that $\mathrm{DM}_{\mathrm{GRB}}$ is typically around $1 \mathrm{pc} \mathrm{cm}^{-3}$ for the ISM afterglow model and $10 \mathrm{pc} \mathrm{cm}{ }^{-3}$ for the wind afterglow model $^{4}$. Based on these results, we cautiously adopt $\sigma_{\mathrm{GRB}}=10 \mathrm{pc} \mathrm{cm}^{-3}$.

- The value of $\mathrm{DM}_{\text {Host }}$ depends on many factors, such as the type of GRB host galaxy, the site of GRB in the host galaxy, the inclination angle of the disk with respect to line of sight, and so on. Based on the DM dispersion of Milky Way, one may expect the uncertainty of $\mathrm{DM}_{\text {Host }}$ could be from tens to hundreds of $\mathrm{pc} \mathrm{cm}^{-3}$. Here we take $\sigma_{\text {Host }}$ as a free parameter. Note that both $\sigma_{\mathrm{GRB}}$ and $\sigma_{\text {Host }}$ should be redshifted.

- The uncertainty $\Delta\left(\mathrm{DM}_{\mathrm{IGM}}\right)$ due to inhomogeneity of the baryon matter in the IGM is an unknown parameter. Numerical simulations (McQuinn 2014) gave a standard deviation $\sigma_{\text {IGM }} \sim 100-$ $400 \mathrm{pc} \mathrm{cm}{ }^{-3}$ around the mean value $\left\langle\operatorname{DM}_{\mathrm{IGM}}(z)\right\rangle$ at $z=0.5-1$. If so, the IGM inhomogeneity effect would be the dominant component for $\mathrm{DM}_{\text {IGM }}$ uncertainty. Without any observational guide, we introduce an unspecified $\sigma_{\operatorname{IGM}}(z)$ to describe this uncertainty.

Given a certain set of cosmological model parameters $\left(H_{0}, \Omega_{b}, f_{\mathrm{IGM}}, \Omega_{\mathrm{M}}, \Omega_{\mathrm{DE}}, \Omega_{\mathrm{k}}, \chi_{\mathrm{e}, \mathrm{H}}(z)\right.$, and $\left.\chi_{\mathrm{He}, \mathrm{H}}(z)\right)$, one can calculate $\left\langle\mathrm{DM}_{\mathrm{IGM}}\right\rangle$ precisely. However, if one infers this value from the observed $\mathrm{DM}_{\mathrm{obs}}$ (Eq.(2)), one would have to incorporate a total uncertainty of (in unit of $\mathrm{pc} \mathrm{cm}^{-3}$ )

$$
\begin{aligned}
\sigma_{\left\langle\mathrm{DM}_{\mathrm{IGM}}\right\rangle} & =\sigma_{\mathrm{obs}}+\frac{\sigma_{\mathrm{GRB}}+\sigma_{\mathrm{Host}}}{1+z}+\sigma_{\mathrm{MW}}+\sigma_{\mathrm{IGM}}(z) \\
& =\frac{10+\sigma_{\mathrm{Host}}}{1+z}+33.34+\sigma_{\mathrm{IGM}}(z)
\end{aligned}
$$

\section{TESTING CAPABILITY OF FRB/GRB SYSTEMS TO CONDUCT COSMOGRAPHY}

We perform Monte Carlo simulations to test how well FRB/GRB systems can be used to constrain the dark energy equation of state. To do so, we need to assume

\footnotetext{
3 http://www.atnf.csiro.au/research/pulsar/psrcat/

4 Note that for extreme parameters in the wind model, DM $\mathrm{GRB}_{\mathrm{G}}$ could reach 100 (Deng \& Zhang 2014). However, such cases could be identified through afterglow modeling and dropped out from the sample.
} 

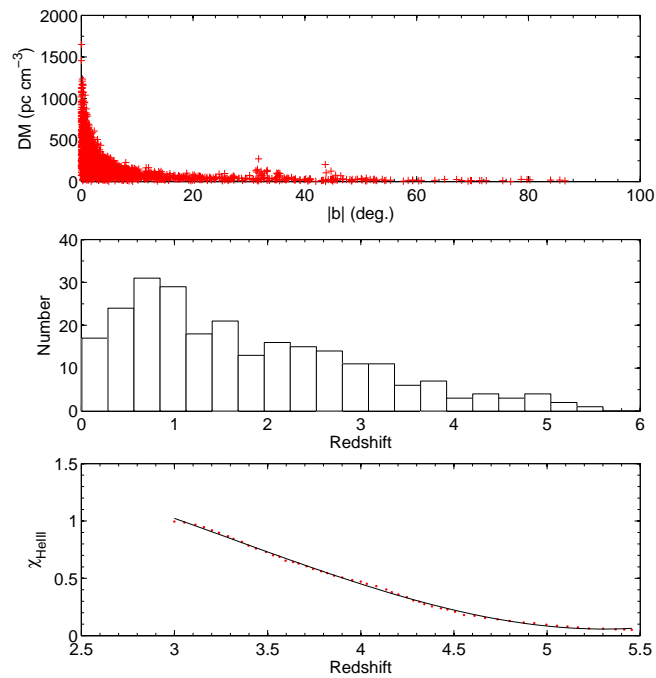

FIG. 1.- a): Measured DM for known pulsars in Milky Way against their Galactic latitude; b) The observed GRB redshift distribution; c) The $\chi_{\text {HeIII }}(z)$ evolution history from numerical simulations of McQuinn et al. (2009) (red dots, their D1 model) and our analytical approximation (solid line).

an underlying cosmological model (i.e. effectively fix a set of cosmological parameters), and then simulate a sample of FRB/GRB systems each with an assigned $z$ and $\left\langle\mathrm{DM}_{\mathrm{IGM}}\right\rangle(z)$. For the $z$-distribution, since no observed FRB/GRB system could be used as a reference ${ }^{5}$, we simulate the $z$-distribution of our sample based on the observed $z$ distribution of the observed $\mathrm{GRBs}^{6}$ (see Fig 1b). For each GRB with an assigned $z$, we calculate its $\left\langle\mathrm{DM}_{\mathrm{IGM}}\right\rangle(z)$ based on Eq.(3), and then assign a $\mathrm{DM}_{\mathrm{IGM}}$ value through introducing the scatter $\sigma_{\left\langle\mathrm{DM}_{\mathrm{IGM}}\right\rangle}$ defined by Eq.(4).

To make use of Eq.(3), we need to assign certain values to relevant parameters. Since $\mathrm{DM}_{\mathrm{IGM}}$ is linearly proportional to $H_{0}, \Omega_{b}$ and $f_{\mathrm{IGM}}$, these three parameters have to be constrained independently for our purpose. Incidentally, these parameters can be constrained independent of the dark energy models. The Hubble constant $H_{0}$ can be constrained using the conventional extragalactic distance scale, while $\Omega_{b}$ can be constrained by CMB or Big Bang nucleosynthesis (BBN) data. Here we adopt the following "benchmark" values recently derived from the joint Plank $+W M A P$ data Hinshaw et al. 2013; Ade et al. 2013) in our simulations: $H_{0}=(67.3 \pm$ 1.2) $\mathrm{km} \mathrm{s}^{-1} \mathrm{Mpc}^{-1}$ and $\Omega_{b}=0.0487 \pm 0.002$. The value of $f_{\text {IGM }}$ is more uncertain. According to the baryon mass summation results of Fukugita et al. (1998), one could derive an estimation of $f_{\text {IGM }} \sim 0.83$ (Deng \& Zhang 2014). Recent simulation results show that for redshifts $z \leq 0.4$, the collapsed phase (galaxies, groups, clusters, etc.) contains $18 \% \pm 4 \%$ baryon mass, which gives $f_{\text {IGM }} \sim 0.82 \pm 0.04$ (Shull et al. 2012). Here we suggest to adopt $f_{\text {IGM }} \sim 0.83$ as the prior. In principle, the mean

5 The two reported candidate FRB/GRB systems (Bannister et al. 2012) unfortunately did not have redshift measurements.

6 The data was collected from an online catalog listed at http://lyra.berkeley.edu/grbox/grbox.php value of the product $\Omega_{b} f_{\text {IGM }}$ could also be directly measured with a large sample of nearby FRB/GRB systems in the future (Deng \& Zhang 2014).

It should be safe to assume $\chi_{\mathrm{e}, \mathrm{H}}(z)=\chi_{\mathrm{e}, \mathrm{He}}(z)=1$ for nearby FRB/GRB systems at $z<3$, since both $\mathrm{H}$ and $\mathrm{He}$ are expected to be fully ionized (Fan et al. 2006; McQuinn et al. 2009). However, to test dark energy equation of state and its dynamical evolution of dark energy $w(z)$, samples with larger redshifts are essential. More accurate expressions for $\chi_{\mathrm{e}, \mathrm{H}}(z)$ and $\chi_{\mathrm{e}, \mathrm{He}}(z)$ are required to reduce the uncertainty. At $z>6$ hydrogen reionization becomes important while the reionization history is poorly known. We suggest that in the future one should use FRB/GRB systems at $z<6$ to perform cosmography studies. In this redshift range, one can approximately take $\chi_{\mathrm{e}, \mathrm{H}}(z)=1$. The fraction of electrons in He atoms that has been ionized should be $\chi_{\mathrm{e}, \mathrm{He}}(z)=$ $(1 / 2) \chi_{\mathrm{HeII}}(z)+\chi_{\mathrm{HeIII}}(z)=(1 / 2)\left(1+\chi_{\mathrm{HeIII}}(z)\right)$, since the ionization energy of $\mathrm{HeI}$ is close to that of $\mathrm{H}$, one may assume that HeI is also fully ionized. McQuinn et al. (2009) have studied HeII ionization history through detailed simulations. For easy application, we fit their numerical results (D1 model in McQuinn et al. (2009)) with a polynomial, so that the mean value of $\chi_{\mathrm{e}, \mathrm{He}}(z)$ can be approximated as

$\chi_{\mathrm{e}, \mathrm{He}}(z)=\left\{\begin{array}{lr}1, & z<3 \dot{3} \\ 0.025 z^{3}-0.244 z^{2}+0.513 z+1.006, & z>3 ;\end{array}\right.$

Such an analytical approximation has a $\sim 4 \%$ error with respect to the numerical results (Fig.1c), and the simulation results are also slightly model-dependent. Nonetheless, in view of the $1 / 8$ coefficient of $\chi_{\mathrm{e}, \mathrm{He}}(z)$ in Eq.(3), these uncertainties are negligible for our purpose.

After fixing the above parameters, we want to test how simulated mock data constrain the underlying dark energy models. We assume an underlying flat $\Lambda \mathrm{CDM}$ model with $\Omega_{\mathrm{M}}=0.315$ and $\Omega_{\Lambda}=0.685$, but introduce a general set of $w \mathrm{CDM}$ models and apply the mock data to check how well the data can reproduce the underlying model.

Figure 2 shows the theoretical $\left\langle\mathrm{DM}_{\mathrm{IGM}}\right\rangle$ (red curves) and distance modulus $\mu$ (blue curves) as a function of $z$ for three $w \mathrm{CDM}$ models $\left(\Omega_{\mathrm{k}}=0, w\right.$ is constant, not evolving with $z$ ): $w=-1$ (solid), $w=-1.2$ (dashed), and $w=-0.8$ (dash-dotted). Overplotted are the simulated $50 \mathrm{FRB} / \mathrm{GRB}$ systems and the observed Union 2.1 SN Ia sample (Suzuki et al. 2012). From this plot, it is clearly seen that the DM curves have a wider separation than the $\mu$ curves to allow an easier differentiation among the models. This is especially so at high redshifts. While all SN Ia are at $z<2$, GRBs have been detected at redshifts as high as $z=8.2$ (Tanvir et al. 2009; Salvaterra et al. 2009). Since FRBs are typically bright, with a peak flux at multi-Jansky level. A moderately large radio telescope with rapid slewing capability would lead to detection of FRBs following GRBs in the redshift range $2<z<6$. As a result, FRB/GRB systems may be a viable way to constrain dark energy equation of state.

To see this point more clearly, we show contour constraints in the $\Omega_{\mathrm{M}}-w 2$-dimensional plane. For simplicity, we take $\sigma_{\operatorname{IGM}}(z)$ as a constant. We first fix the uncertainties of host galaxy and IGM inhomogeneity as $\left(\sigma_{\text {Host }}, \sigma_{\mathrm{IGM}}\right)=(30,50)$, and generate three samples 


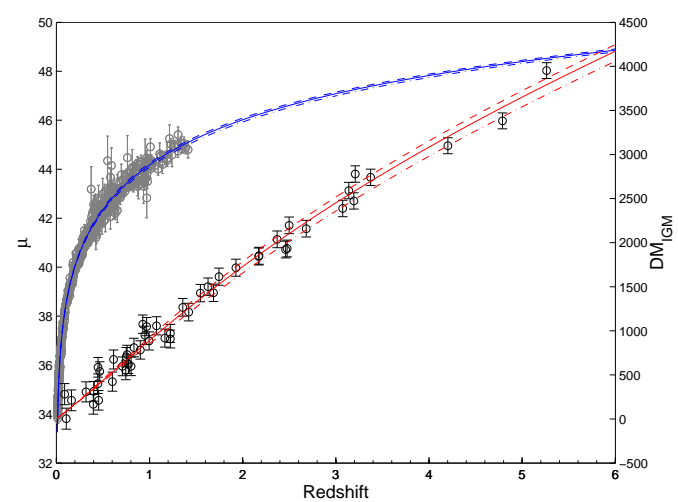

FIG. 2.- Hubble Diagram and $\left\langle\mathrm{DM}_{\mathrm{IGM}}\right\rangle$ evolution for different $w \mathrm{CDM}$ models (a flat universe is assumed here and $\Omega_{m}$ is fixed at 0.315 ). The solid lines are for $w=-1$, dash lines are for $w=-1.2$, and the dash-dot lines are for $w=-0.8$. Grey circles are for Union 2.1 SN Ia sample (Suzuki et al. 2012), and black circles show one example of our simulated FRB $/$ GRB sample $\left(\mathrm{N}_{\mathrm{sam}}=50, \sigma_{\text {Host }}=\right.$ $30 \mathrm{pc} \mathrm{cm}^{-3}$ and $\left.\sigma_{\mathrm{IGM}}=50 \mathrm{pc} \mathrm{cm}^{-3}\right)$.

with $\mathrm{N}_{\text {sam }}=30,60,100$, respectively ${ }^{7}$. Figure 3 a shows the contour contrast for different sample sizes. For comparison, we also plot the contours by applying the Union $2.1 \mathrm{SN}$ Ia data. One can see that with a moderate sample size of several 10s, the contour size of FRB/GRB systems is already comparable to that of SN Ia (which has more than 500 SN Ia). Furthermore, when combining FRB/GRB systems with SN Ia, a much better constraint is achieved. This is mainly due to the fact that FRB/GRB systems are distributed in a much wider redshift range towards high- $z$, where better constraints on the models can be achieved. Our results are generally consistent with Zhou et al. (2014).

We also test the effects from uncertainties of the host galaxy and inhomogeneity. In this case, we fix our sample number as 60 and generate three samples with $\left(\sigma_{\text {Host }}, \sigma_{\text {IGM }}\right)=(30,50),(30,200),(100,50)$, respectively. As shown in Figure $3 \mathrm{~b}$, the results are more sensitive to $\sigma_{\text {IGM }}$ than $\sigma_{\text {Host }}$. This is because the latter becomes less significant at high redshifts due to the $(1+z)$ factor, while the high redshift data are more powerful to differentiate among the models.

\section{4. $D_{\mathrm{L}}(Z)$ VS. $\left\langle\mathrm{DM}_{\mathrm{IGM}}\right\rangle(Z)$ AND IMPLICATION}

"Standard candles" make use of the luminosity distance of the source, $D_{\mathrm{L}}(z)=c H_{0}^{-1}(1+z) \int_{0}^{\mathrm{z}} \frac{d z^{\prime}}{E\left(z^{\prime}\right)}$. Comparing with Eq.(3), one has

$$
\frac{D_{\mathrm{L}}(z)}{\left\langle\mathrm{DM}_{\mathrm{IGM}}\right\rangle(z)}=\frac{8 \pi G m_{p}}{3 H_{0}^{2} \Omega_{b} f_{\mathrm{IGM}}} \frac{(1+z) \int_{0}^{z} \frac{d z^{\prime}}{E\left(z^{\prime}\right)}}{\int_{0}^{z} \frac{\chi\left(z^{\prime}\right)\left(1+z^{\prime}\right) d z^{\prime}}{E\left(z^{\prime}\right)}} .
$$

For given $H_{0}, \Omega_{b}$ and $f_{\text {IGM }}$ values (measured independently), different dark energy cosmology models are contained in the expression of $E(z)$, which is essentially canceled out in the $D_{\mathrm{L}}(z) /\left\langle\mathrm{DM}_{\mathrm{IGM}}\right\rangle(z)$ ratio. This ratio is expected to only weakly depend on dark energy models.

\footnotetext{
7 These sample sizes are adopted according to a realistic estimate of possible FRB/GRB systems that might be detected in a decade time scale, based on the detection rate derived from true searches (Bannister et al. 2012) and GRB data analysis (Lü \& Zhang 2014).
}
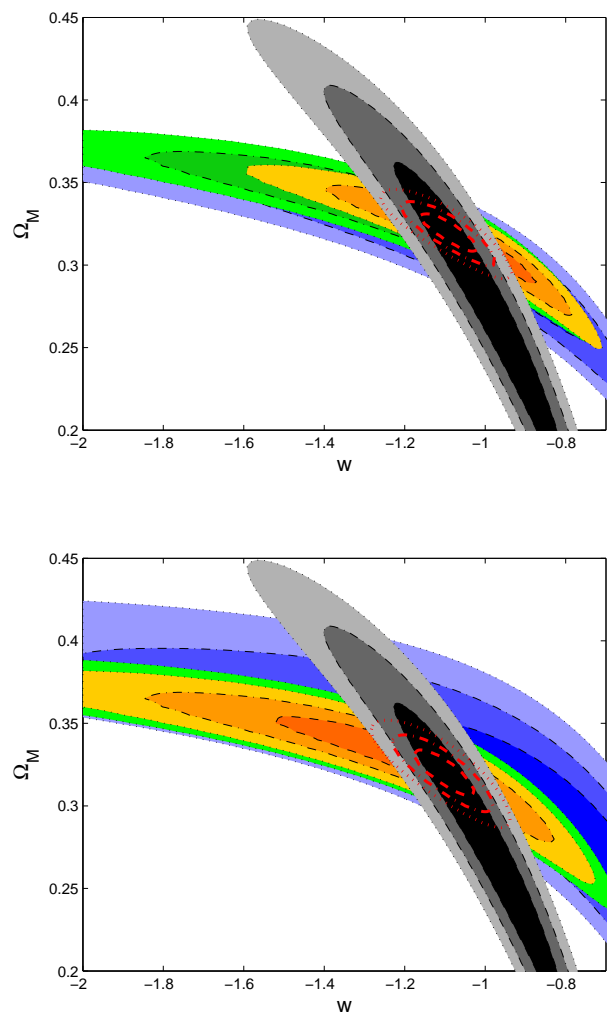

FIG. 3.- Constraint results for $w \mathrm{CDM}$ models using simulated FRB/GRB systems, compared with Union 2.1 SN Ia results (grey contours): (a) effect of different sample sizes: $N_{\text {sam }}=30$ (blue contours), 60 (green contours), and 100 (orange contours). Other parameters are fixed to $\sigma_{\mathrm{Host}}=30, \sigma_{\mathrm{IGM}}=50 ;(\mathrm{b})$ effect of $\sigma_{\mathrm{Host}}$ and $\sigma_{\text {IGM }} . N_{\text {sam }}$ is fixed to $60: \sigma_{\text {Host }}=30, \sigma_{\text {IGM }}=200$ (blue contours), $\sigma_{\mathrm{Host}}=100, \sigma_{\mathrm{IGM}}=50$ (green contours), and $\sigma_{\text {Host }}=$ $30, \sigma_{\text {IGM }}=50$ (orange contours).

To verify this, we again take the $w$ CDM cosmology models as an example. We choose a relatively large parameter space with $-1.2<w<-0.8$ and $0.2<\Omega_{\mathrm{M}}<0.4$. As shown in Figure 4 , the $D_{\mathrm{L}} / \mathrm{DM}_{\mathrm{IGM}}$ ratio only differs by less than $1 \%$ with respect to the $\Lambda \mathrm{CDM}$ model at $z<6$, which is indeed negligible.

This insensitivity of the $D_{\mathrm{L}}(z) /\left\langle\mathrm{DM}_{\mathrm{IGM}}\right\rangle(z)$ ratio on the dark energy models makes it convenient to combine standard candles (e.g. Type Ia SNe) and FRB/GRB pairs to conduct cosmography. While the theoretical values of $\left\langle\mathrm{DM}_{\mathrm{IGM}}\right\rangle(z)$ are well defined, determining them from the data is not easy, which requires to accumulate a large enough sample of FRB/GRB samples in many redshift bins to cancel out the inhomogeneity effect from different lines of sight. The insensitivity of the $D_{\mathrm{L}}(z) /\left\langle\mathrm{DM}_{\mathrm{IGM}}\right\rangle(z)$ ratio allows one to easily determine the shape of $\left\langle\mathrm{DM}_{\mathrm{IGM}}\right\rangle(z)$ based on the well-mapped $D_{\mathrm{L}}(z)$ from the SN Ia data (regardless of the dark energy models). Even though the normalization of $\left\langle\mathrm{DM}_{\mathrm{IGM}}\right\rangle(z)$ depends on $H_{0}, \Omega_{b}$, and $f_{\text {IGM }}$, by knowing the shape of $\left\langle\mathrm{DM}_{\mathrm{IGM}}\right\rangle(z)$ one can combine $\mathrm{FRB} / \mathrm{GRB}$ systems at all redshifts to "calibrate" $\left\langle\mathrm{DM}_{\mathrm{IGM}}\right\rangle(z)$ and find out the normalization. This requires a much smaller sample to achieve the calibration purpose. With $\left\langle\operatorname{DM}_{\text {IGM }}\right\rangle(z)$ well mapped, one can then directly study the scatter of 


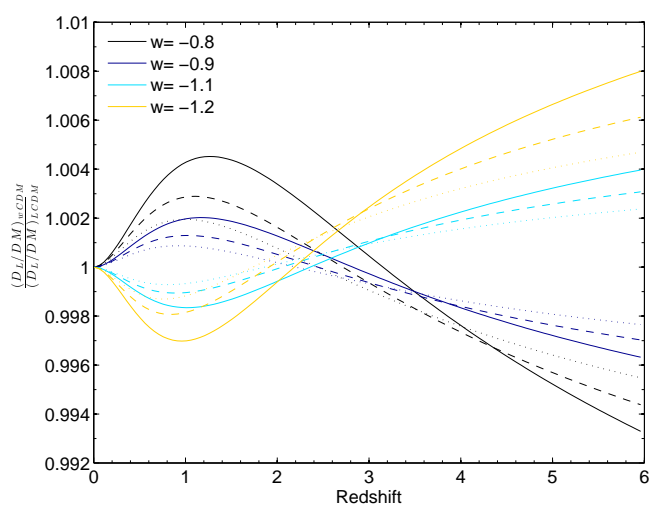

FIG. 4.- The $D_{\mathrm{L}}(z) /\left\langle\mathrm{DM}_{\mathrm{IGM}}\right\rangle(z)$ ratio in different $w \mathrm{CDM}$ models $(w=-0.8,-0.9,-1.1,-1.2)$ normalized to that of the $\Lambda \mathrm{CDM}$ model. For each model, three $\Omega_{\mathrm{M}}$ values are adopted: 0.2 (solid), 0.3 (dashed), 0.4 (dotted).

$\mathrm{DM}_{\mathrm{ICM}}$ due to local IGM inhomogeneity (e.g. McQuinn 2014) as well as its redshift evolution (i.e. $\sigma_{\mathrm{IGM}}(z)$ ).

\section{SUMMARY AND DISCUSSION}

FRB/GRB systems, if confirmed to be commonly exist, have great potential to infer cosmological parameters, especially to constrain the equation of state of dark energy. We have shown that with a moderate sample size of several 10s, one may reach a constraint on $w$ comparable to a large SN Ia sample. Combining SN Ia data and FRB/GRB systems, one may achieve unprecedented accuracy in constraining $w$. The insensitivity of the $D_{\mathrm{L}}(z) /\left\langle\mathrm{DM}_{\mathrm{IGM}}\right\rangle(z)$ ratio offers the advantage of using a relatively small sample to cabibrate $\left\langle\mathrm{DM}_{\mathrm{IGM}}\right\rangle(z)$ and to diagnose the local IGM inhomogeneity as well as its redshift distribution.

Our method is also applicable if the redshifts of FRBs can be measured independently with other methods. Zhou et al. (2014) explored this possibility and reached the similar conclusion that FRBs can constrain dark energy equation of state and IGM inhomogeneity. FRB/GRB associations provide a practical method to measure redshifts of FRBs, and the sample size adopted in this paper is based on observational and theoretical insights of GRBs as well as the results of preliminary searches of FRB/GRB associations (Bannister et al. 2012).

In the end, we want to point out some caveats of our method:

1) If most of the FRBs (or even all of them) are eventually proved to be of a galactic origin, or the redshifts of FRBs could never be measured, FRBs are no longer relevant for cosmography.

2) As shown in our simulations, the constraint results for cosmological parameters are most sensitive to the intrinsic IGM inhomogeneity $\sigma_{\text {IGM. }}$. Even though the calibration of $\left\langle\mathrm{DM}_{\mathrm{IGM}}\right\rangle$ could be achieved (see section 4), if $\sigma_{\text {IGM }}$ is very large, its uncertainty could still reduce the constraint accuracy dramatically. A (much) larger sample size than simulated here is needed to make this method competitive.

3) The GRB hosts likely evolve with redshift (e.g. Perley et al. 2013). This suggests that both $\mathrm{DM}_{\text {host }}$ and $\sigma_{\text {host }}$ could be redshift dependent. In our simulations, we adopt a constant free parameter for $\sigma_{\text {host }}$, and its contribution to $\sigma_{\mathrm{DM}} \mathrm{IGM}_{\mathrm{IG}}$ is reduced at higher redshifts due to $(1+z)$ factor (Eq. (4). If it turns out that $\mathrm{DM}_{\text {host }}$ and $\sigma_{\text {host }}$ would increase with redshift and $\sigma_{\text {host }}$ could become comparable or even larger than $\sigma_{\mathrm{IGM}}(z)$, the accuracy of our results could be reduced dramatically. To keep the method competitive, either a larger sample is required, or more observational information about the GRB host galaxy population is needed.

4) If the dispersions of various parameters is intrinsically non-Gaussian, a larger sample than simulated may be needed to quantify these dispersions to achieve an accurate constraint on dark energy models.

We thank Fa-Yin Wang for a helpful discussion. This work is partially supported by National Basic Research Program ("973" Program) of China under Grant No. 2014CB845800, the NSFC (11273005) and SRFDP (20120001110064). HG acknowledges a Fellowship from China Scholarship Program for support.

\section{REFERENCES}

Ade, P. A. R., Aghanim, N., et al. 2013, arXiv:1303.5076

Amati, L. 2013, The Astronomical Review, 8, 010000

Anderson, L., Aubourg, E., Bailey, S., et al. 2012, MNRAS, 427, 3435

Bannister, K. W., Murphy, T., Gaensler, B. M., \& Reynolds, J. E. 2012, ApJ, 757, 38

Barkana, R., \& Loeb, A. 2004, ApJ, 601, 64

Beutler, F., Blake, C., Colless, M., et al. 2011, MNRAS, 416, 3017

Bannister, K. W., Murphy, T., Gaensler, B. M., \& Reynolds, J. E. 2012, ApJ, 757, 38

Bannister, K. W., \& Madsen, G. J. 2014, MNRAS, 440, 353

Chevallier, M., \& Polarski, D. 2001, International Journal of Modern Physics D, 10, 213

Cucchiara, A., Levan, A. J., Fox, D. B., et al. 2011, ApJ, 736, 7

Dai, Z. G., Liang, E. W., \& Xu, D. 2004, ApJ, 612, L101

Deng, W., \& Zhang, B. 2014, ApJL, in press, arXiv:1401.0059

Falcke, H., \& Rezzolla, L. 2014, A\&A, in press, arXiv:1307.1409

Fan, X., Carilli, C. L., \& Keating, B. 2006, ARA\&A, 44, 415

Fukugita, M., Hogan, C. J., \& Peebles, P. J. E. 1998, ApJ, 503, 518

Ghirlanda, G., Ghisellini, G., Lazzati, D., \& Firmani, C. 2004, ApJ, 613, L13

Hinshaw, G., Larson, D., Komatsu, E., et al. 2013, ApJS, 208, 19
Ioka, K. 2003, ApJ, 598, L79

Kulkarni, S. R., Ofek, E. O., Neill, J. D., Zheng, Z., \& Juric, M. 2014, arXiv:1402.4766

Liang, E., \& Zhang, B. 2005, ApJ, 633, 611

Linder, E. V. 2003, Physical Review Letters, 90, 091301

Loeb, A., Shvartzvald, Y., \& Maoz, D. 2014, MNRAS, 439, L46

Lorimer, D. R., Bailes, M., McLaughlin, M. A., Narkevic, D. J., \& Crawford, F. 2007, Science, 318, 777

Lü, H.-J., \& Zhang, B. 2014, ApJ, submitted, arXiv:1401.1562

Manchester, R. N., Hobbs, G. B., Teoh, A., \& Hobbs, M. 2005, AJ, 129, 1993

McQuinn, M., Lidz, A., Zaldarriaga, M., Hernquist, L., \& Dutta, S. 2008, MNRAS, 388, 1101

McQuinn, M., Lidz, A., Zaldarriaga, M., et al. 2009, ApJ, 694, 842 McQuinn, M. 2014, ApJ, 780, L33

Perley, D. A., Levan, A. J., Tanvir, N. R., et al. 2013, ApJ, 778, 128

Riess, A. G., Filippenko, A. V., Challis, P., et al. 1998, AJ, 116, 1009

Rowlinson, A., O'Brien, P. T., Metzger, B. D., Tanvir, N. R., \& Levan, A. J. 2013, MNRAS, 430, 1061

Salvaterra, R. et al. 2009, Nature, 461, 1258

Schaefer, B. E. 2007, ApJ, 660, 16 
Shull, J. M., Smith, B. D., \& Danforth, C. W. 2012, ApJ, 759, 23 Suzuki, N., Rubin, D., Lidman, C., et al. 2012, ApJ, 746, 85

Tanvir, N. R., Fox, D. B., Levan, A. J., et al. 2009, Nature, 461, 1254

Taylor, J. H., \& Cordes, J. M. 1993, ApJ, 411, 674

Thornton, D., Stappers, B., Bailes, M., et al. 2013, Science, 341, 53

Virgili, F., Zhang, B., Nagamine, K. \& Choi, J.-H. 2011, MNRAS, 417,3025
Wang, F. Y., Bromm, V., Greif, T. H., Stacy, A., Dai, Z. G., Loeb, A. \& Cheng, K. S. 2012, ApJ, 760, 27

Zhang, B. 2014, ApJ, 780, L21

Zhou, B., Li, X., Wang, T., Fan, Y.-Z., \& Wei, D.-M. 2014, arXiv:1401.2927 